Wolfgang $\mathrm{H}$ aubrichs

\title{
APPELLATIV UND ORTSNAME IN ÄLTERER ZEIT. DREI FALLSTUDIEN
}

\begin{abstract}
„Ältere Zeit" im Titel dieses Aufsatzes ist zweifellos ein Euphemismus, denn die Zeit, auf die sich mein Interesse richtet, ist die Zeit der Merowinger und Karolinger, ist die Zeit des frühen Mittelalters. Auch wenn ich manche relativ junge Quellen aus dem hohen Mittelalter, der F rühen N euzeit oder gar rezente Flurnamen und Dialekte rekonstruierend benutze, geschieht dies nur in der Absicht, durch den dunklen Berg der Zeit einen Stollen zu treiben, und dann nicht darin steckenzubleiben, sondern irgendwo dahinter in eine blaue Lagune der Frühzeit, in einen Wörtersee der Vergangenheit einzulaufen. Man weiß natürlich längst, und es ist längst mehrfach erprobt (D it t maier 1963; P ost 1982; R amge 1987; 2002), dass die Namenwelt des europäischen Kulturkreises aus Lexemen des W ortschatzes der Allgemeinsprache besteht, und dass, wenn man Methoden der historischen Sprachforschung, der Onomastik, gegebenenfalls auch der Interferenzforschung anwendet (z. B. H aubrichs/R amge 1983), und wenn man dazu noch das Glück einer günstigen Quellenlage hat, man aus den Toponymen, den Ortsnamen also, A ppellative, die einstmals in sie eingingen, wieder herauspräparieren kann, so wie manchmal ein Insekt aus dem Bernstein. Da dem so ist, wird die Namenforschung für die Rekonstruktion von Sprachen oder Dialekten überall dort interessant, wo wir nicht über genügend Textquellen verfügen, und das ist für die Zeit vor dem neunten J ahrhundert (genau genommen aber noch bis ins späte Mittelalter) doch sehr der F all.

Onomastik bewährt sich als Hilfsdisziplin, Hilfswissenschaft - und welches vornehmere Ziel lässt sich formulieren als das eines Wissens, das helfen kann - als Hilfsdisziplin vor allem der Sprachforschung, der Kulturgeschichte, ja sogar - in diesen frühen Zeiten - der Geschichte, vor allem der Geschichte ethnischer und sprachlicher Akkulturation. Sie bewährt sich in der Rekonstruktion von Trümmersprachen, wenig bekannter Dialekte, regionaler Interferenzen von Sprachen, in der Erforschung von Son-
\end{abstract}


dersprachen bestimmter Domänen - z. B. des Rechts, der agrarischen Welt, der Welt des Reisens und der W ege usw.

Einige von diesen Möglichkeiten der Rekonstruktion werde ich an nur vier Beispielen zu zeigen versuchen, die ein geheimes Zentrum der Konvergenz besitzen, das - wie ich hoffe - nach dieser einleitenden Anpreisung und nach der Sachanalyse aus dem Labyrinth der Belege hervortreten wird.

\section{A mt sbezeichnungen in Ortsnamen}

Amtsbezeichnungen kommen in Ortsnamen, vor allem auch in Siedlungsnamen, durchaus häufiger vor (vgl. Haubrichs 2003). Das erklärt sich ebenso wie bei den mit Personennamen komponierten Toponymen etwa vom Typ a. 780/802 Leidmares-heim (Leimersheim, Pfalz, Kr. Germersheim) zum PN ahd. Leid-mar (D ol ch/Gr eul e 1991, S. 285). Diese Siedlungsnamen bewahren $\mathrm{H}$ inweise auf den Gründer oder einen frühen Besitzer. Bei den Amtsbezeichnungen darf man in Analogie dazu erwarten, dass sich aus innen auf ehemaliges Amtsgut als Benennungsmotiv schließen lässt. In nicht wenigen Fällen lässt sich dies beweisen, so etwa im Falle von

(1) Bischmisheim (Stadt Saarbrücken), a. 884 Biscofesheim, Ende 9. J h. Piscofesheim ,Siedlung des Bischofs', in diesem Falle Fernbesitz des Bischofs von Reims, der nach a. 600 zu einer vom älteren königlichen Fiskus Berna (Behren bei Forbach, F, Dép. Moselle), den der Bischof erworben hatte, ausgehenden Siedlungsgründung führte ( $\mathrm{H}$ aubrichs/St ein 1999, S. 126ff.).

Auch in der Pfalz, in Franken und im Elsass lassen sich solche F älle speziell bischöflicher Gründungen nachweisen, deren Benennungsanlass im Siedlungsnamen konserviert wurde, z. B. nicht allzu weit von Straßburg entfernt das dem Straßburger Bischof gehörige.

(2) Bischheim (F, Dép. Bas-Rhin), a. 662 Fä., 12. J h. (angebliche Schenkung Dagoberts II. an die Straßburger Kirche, deren echter Kern freilich der Besitztitel war) Bischovisheim, a. 1116 Biscofesheim marcha (B r uckner 1949, Nr. 42; R eichsl and III, S. 97).

Besonders interessant für Landes- und Reichsgeschichte wird es, wenn sich die Bezeichnung des obersten Amtsträgers eines regnum und nominalen Besitzers des Staatslandes, des Fiskus, in Ortsnamen abbildet, die Bezeichnung kuning also, die im Althochdeutschen, aber wohl auch schon vorher in den Reichen der Franken, aber auch der Langobarden mit dem lateinischen Amtstitel rex identisch war (M eid 1966, S. 186ff.). In zahlrei- 
chen Fällen lässt sich auch hier der Zusammenhang zwischen Orten vom Typ *K uninges-hoven ,Königshofen' oder *K uninges-haima ,K önigsheim" mit überliefertem Königsgut, d. h. Fiskalgut herstellen, z. B. in den im 7. und 8. J ahrhundert intensiv vom Königtum geprägten ostfränkischen Landen um den Main:

(3) (Bad) K önigshofen im Grabfeld (Kr. Rhön-Grabfeld). a. 822 Chuningishaoba, a. 889 de fiscis dominicis id est de [....] Chuningeshofa [....] in his fiscis et villis dominicis <*K uningeshufa ,H of des Königs' (R eit zenst ein 1986, S. 51).

Am Mittelrhein wurde gar ein ganzer Bezirk als Königsgut aus dem Rheingau um Wiesbaden als Sondereigen herausgeschnitten und entsprechend benannt:

(4) Königssondergau, 9. J h. Kuningessundra ,Sondergut des Königs'; a. 909 kop. Cuningishuntra ,Centena des Königs' (P ol enz 1961, S. 74, 169, 285).

Im Elsass sind mit ahd. kuning zusammengesetzte Toponyme relativ häufig vertreten. Ein den Zusammenhang mit dem Königsgut ad oculos demonstrierender Fall ist Z. B. Kinzheim (F, Dép. Bas-Rhin) bei Schlettstadt/Sélestat, das a. 774 und öfter explizit als fiscus firmiert (Bruckner 1949, Nr. 254, 528, 540, 596, 601, 607; Reichsl and III S. 518, 515; AIbrecht 1891/98: I, Nr. 122, 697; Sitt l er 1958: Nr. 311):

(5) Kinzheim /Dép. Bas-Rhin, Ct. Sélestat): a. 774 or. (Schenkung K arls des Großen an die Abtei Leberau/Lièvre [....] ex marca fisco nostro Quvningishaim ; a. 843 or. (Schenkung Lothars I.) [.... villam quandam, que K unigesheim nominatur [....]; a. 854 kop. (Bestätigung Lothars I. für die Abtei Leberau/Lièvre) [....] exmarcka fisci praedicti domni Karoli [Karls des Großen], qui Qıvningishaim dicitur [....]; a. 877 or. (Schenkung der elsässischen Besitzungen, die ihr der Neffe Lothar II. gegeben hatte, durch Äbtissin Berhta an ihre Abtei St. Felix und Regula in Zürich) Chuningesheim; a. 878 or (Bestätigung der Schenkungen Lothars II. für St. Felix und Regula durch Karl III.) Cunigesheim; a. 881 kop. (Ertauschung elsässischer Güter vom Hochstift Chur durch Karl III.) [....] in villis nominatis Sclectistat [Schlettstadt], Chuniggesheim [....]; \pm a. 1230 Regis villa; a. 1298 kop. Künßheim; a. 1367 or. de K önishein; a. 1404 or. Kunsheim; mda. Kinze.

Sprachlich ist an diesem mit dem merowingischen SiedlungsnamenGrundwort *-haima- - zusammengesetzten Toponym noch bemerkenswert, dass er germ. [ai], das im frühen 8. J ahrhundert althochdeutsch zu [ei] wird, in den frühesten Belegen noch festhält, so dass eine ältere V orlage nicht ausgeschlossen erscheint, und dann, dass das doppelt ausgefertigte Originaldiplom Karls des Großen von a. 774, das zweifellos von einem romanischen Schreiber geschrieben wurde, mit romanischer Graphie < qu > germ. [k] festhielt, was möglich war, da gallorom. [qu] (vgl. frz. qui) bereits zu [k] geworden war. Aus diesem Diplom übernahm a. 854 der 
Schreiber des Diploms Lothars I. seine Schreibform des Namen. Das Originaldiplom der aus königlicher Familie stammenden Äbtissin Berhta aus Zürich zeigt a. 877 die hochalemannische Verschiebung von ahd. [k] > [kch]; das Originaldiplom Karls III. von a. 878 hält die seit spätalthochdeutscher Zeit beginnende Nebenform von kuning mit n-Schwund vor [g] fest.

Noch ein weiterer K önigsortsname aus dem E Isass mag hier angeführt werden. Auf Herzogsbesitz, der letzten Endes wohl auf merowingischen Königsbesitz zurückgeht, urkundet der elsässische, aus dem Etichonenhause stammende Herzog Adalbert a. 722 in einem Vorort von Straßburg, der später Königshofen heißen wird und auf die Namenform frühalthochdeutsch *K uningis-hufa wie das oben behandelte ostfränkische B eispiel zurückgeht, hier aber als curtis Regia villa bezeichnet wird, wobei der Zusatz villa anzeigt, dass wir es bei curtis Regia zweifellos mit einem Ortsnamen, einem sprechenden allerdings, zu tun haben (Bruckner 1949, Nr. 100, 622; R eichsl and III, S. 530):

(6) Königshofen (Straßburg/Strasbourg, Dép. Bas-Rhin): a. 722 kop. (Schenkung Herzog Adalberts an K loster Honau) Actum Stratburgo civitate in curte Regia villa, que est in suburbio Civitatis novo, quam ego ex novo opere construxi [...]; a. 902 Fä. 1260/70 (Schenkung des Grafen Liutfrid und seiner Söhne an Kloster St. Trudpert im Schwarzwald, evtl. mit einem echten K ern) [...] in Norgauwe [elsässischer Nordgau] unam curtim, que dicitur K iunigishova [...]; a. 903 Fä. 13./14. J h. [...] curtim qui vocatur K unigishova [...]; a. 1144 Cungeshoven; a. 1163 Cunegeshoven.

Für die Rekonstruktion verlorener Appellative verdient mehr Beachtung ein Ortsname, der offensichtlich eine seltene fränkische und dann althochdeutsche Bezeichnung von K önigsleuten enthält (K auf mann 1976, S. 126):

(7) Köngernheim westlich Oppenheim (K r. Mainz-Bingen):

a. 800 kop. Cunigernheim, a. 804 kop. Cuningero heim, a. 1194/98 Chungirheim iuxta rivum Selsa [Selz]; a. 1305 K unegernheim <*K uningaro-haima ,Siedlung der Königsleute'

Dem schließt sich in der Nähe bei Alzey an (K auf mann 1976, S. 78f.):

(8) Gau-K öngernheim (K r. Alzey): a. $1268 \mathrm{~K}$ ungern-heim; a. $1299 \mathrm{~K}$ ungernheym; a. $1429 \mathrm{~K}$ yngernheim by Qlernheim <*K uningaro-haima.

In Bayern schließt sich (anscheinend unidentifiziert) ein weiterer Ortsname, diesmal ursprünglicher Gewässername, an (F ör stemann 1911/16, S. 1756f.).

(9) a. 821 Chuningaropah <*K uningaro-baki ,Bach[tal] der K önigsleute'. 
Alle drei Ortsnamen enthalten (im Gen. Flur.) das zu rekonstruierende Appellativum ahd. *Kuning-äri mit dem aus lat. -arius entlehnten Suffix germ. *ārja, ahd. g-āri (H enzen 1957, § 98), das ursprünglich vorwiegend von Substantiven abgeleitete Nomina agentis bildete. Hier dürfte es sich um eine späte analogische Bildung mit patronymischem Sinn ,zum K önig gehörig' handeln, so wie man etwa Müller < ahd. mulināri auch als,zur Mühle gehörig', M ünzer < ahd. munizzāri als ,zur Münze gehörig', Kämme rer < ahd. kamarāri als ,zur camera zum inneren Bezirk eines Großen gehörig' verstehen konnte.

Die Belegung von ausgestorbenem oder schwach belegtem W ortschatz durch Toponyme ist durchaus nicht selten und kann im Einzelfall weitreichende Folgen haben. So beim folgenden Beispiel, das in die Frühzeit des fränkischen Rechts zurückführt.

In der ,Lex Salica' $(\S 46,6)$ wird "dem ligitimus mallus publicus unter dem Vorsitz des thungin der mallus ante regem, das Gericht vor dem K önig" gegenübergestellt (Schmidt-W iegand 1972; 1989, S. 157ff.; 1998; W eitzel 1985, S. 434-446; 1992). Dieser thunginus wird in der ,Lex Salica' $(\S 44,1 ; 46,1)$ durchweg als Vorsitzender des Things oder mallus aufgerufen, der stets das Rechtssymbol des Schildes (scutum) bei sich zu führen hat, das also wohl mit seiner Funktion eng verknüpft war. Tunginus aut centenarius mallo indicant, der thunginus oder der Zentenar (als Vorsteher einer centena oder ahd. hunta ,H undertschaft') sagen das mallum an. Man braucht gar nicht so weit zu gehen, wie es der Historiker Rainer $W$ enskus in einem hypothesenreichen Aufsatz zu dieser frühfränkischen Institution tat (W enskus 1986), um zu erkennen, dass der thunginus eine bedeutsame, möglicherweise regional begrenzte öffentliche F unktion innehatte. Er wird später vom iudex hoc est comes aut grafio ersetzt, verschwindet nach der ,Lex Salica' aus dem fränkischen Recht, so dass man hat vermuten können, dass Chlodwig selbst oder unmittelbare Nachfolger dieses selbständige und nicht vom Königtum abgeleitete Amt beseitigt hätten. Um so bedeutsamer ist es, dass die Amtsbezeichnung des thungIn gleich dreimal im Elsass, und - soweit ich sehe - nur im Elsass vorkommt.

Etymologisch gehört thungПn nach überwiegender Meinung der Forschung zu einem westgermanischen, wahrscheinlich nur fränkischen *pung-inaz, einer A bleitung zu einer A blautform auf [-u-] von germ. *bengan. ,Übereinkommen, Versammlung, Thing', as. thing, ae. ping, ahd. ding, das vermutlich auch im Ortsnamen Düngstrapp (Kr. Wudeshausen), a. 890 Dungasthorpe belegt ist. Vergleichbar ist die ableitende Schwundstufe des Verbum germ. *beng- $\sigma$ - eine Versammlung abhalten, einen Vertrag abschließen, gerichtlich handeln', as. thingon, ahd. dingon (vgl. ae. pingian), 
dessen nhd. Entwicklung dingen im praeteritalen Partizip ge-dungen ja bis heute den Ablaut festhält (M eid 1966, S. 185).

Dem Suffix nach gehört die Bildung germ. *kung-inaz nämlich in eine Gruppe von in den germ. Sprachen gut vertretenen denominalen Bezeichnungen von Ämtern mit Leistungs- und Repräsentationsfunktionen, die, wie W olfgang $M$ eid gezeigt hat, ein $n$-haltiges Suffix verwenden, z. B.

- germ. *peuda-na-z (got. piudans, an. pjóđann, ae. peoden as. thiodan) ,Leiter der peuda, des Volkes; Herrscher';

- germ. *kenp-(i)na-z, got. kindis (romanisiert burg. hendinus) zu *kenpa- ,gens, Sippe, Geschlecht' (an. kind), in der gotischen Bibelübersetzung den römischen Statthalter bezeichnend, ursprünglich aber vielleicht einen ,U nterkönig' bzw. ,Stammesfürsten';

- germ. *druhti-na-z (an. dróttin, ae. dryhten, as. druhtin, ahd. truht】n) ,Gefolgschaftsherr' zu germ. *druhti- ,Gefolgschaft' etc.

Zu diesen denominalen Ableitungen gehört auch *bung(i)naz, frk. thung[n als Basis von frankolateinisch thunginus, dessen regelgerechte W eiterentwicklung ahd. dungin lauten müsste. Genau diese W eiterentwicklung finden wir aber in drei seit dem 8. J ahrhundert belegten elsässischen Siedlungsnamen der merowingischen Schicht auf -haima- (B r uckner 1949, Nr. 208; W al ter 1908, Nr. 249; Reichsl and III, S. 223f.; Sittler 1958: Nr. 202; $\mathrm{Cl}$ auss 1895 , S. 253):

(10) † Dinzheim (Gde. Heilig-K reuz bei Colmar, Dép. Haut-R hin): a. 768 or. (Schenkung des Sigifrid an seinen Sohn Altman) [...] in villa Tunginisheim, qui est in marca Heruncheim, et in ipso fine $\mathrm{H}$ eruncheim marca de silva [...] etc. [...] actum in villa $\mathrm{H}$ eruncheim, publice [...]; a. 1319 or. von Tvngingshein; a. 1335 Tungeshein; a. 1344 Tingensheim; a. 1365 or Tüngenshein; a. 1376 or. Tungeshein; 15. J h. Flurname dingsen weg; mda. Dinzen (F orsthaus) $<*$ Th)unginis-haima

Es ist bemerkenswert, dass a. 768 Tunginishei $m$ nur einen Ort unter anderen innerhalb der größeren Mark von Oberhergheim darstellt. Dort ist a. 742 auch der Grußgrundbesitzer Rantwig, Sohn des Chrodwig, Enkel des Chrodio und Gefolgsmann des etichonischen Herzogs Liutfrid, begütert, wobei inm der Besitz in Heruncovillare von seiten seiner Mutter Oda und seiner Schwester Basilla zukam (GIöckner /D oll 1979, Nr. 52). Die Namenbildung erweist $\mathrm{H}$ eruncovillare, $\mathrm{H}$ eruncheim <*H erunco-haima als ,Siedlung der Herunge' (Gen. Plur. auf -0), so wie Thorenco-haime a. 742 in gleicher Urkunde (heute Türkheim) die ,Siedlung der Thüringe' bedeutet. Es ist an einen kleinen Personenverband zu denken, wie wir inn auch in Schwaben mit den Pleonungen und in der Franche-Comté mit den Skutingen und Warasken vorfinden. Im Zusammenhang mit dem Amt des thunginus ist festzuhalten, dass a. 768 das placitum, auf dem die Schenkung 
verhandelt wird, in eben jener marca stattfindet, obwohl die Schenkung noch neun weitere Orte betrifft.

W eitere mit *thung】n komponierte Siedlungsnamen finden sich mit

(11) Dingheim bei Truchtersheim (F, Dép. Bas-R hin): a. 788 kop. 9. J h. (Schenkung des Voto in 15 Orten des Elsass an Fulda) [...] in Tunchinashaim [...]; a. 1116 Dungenesheim; a. 1141 Ti[n]gensheim; a. 1226 Tungensheim; a. 1253 Tungensheim; $a .1279$ Dungensheim; a. 1297 Digensheim; a. 1301 Dingesheimb; a. 1347 Tuenngensheim; a. 1355 Dengesheim; mda. Dense, Dingse <*T(h)unginis-haima (Bruckner 1949, Nr. 176; Reichsl and III, S. 223; $\mathrm{Cl}$ auss 1895. $\mathrm{S} 252$ ).

(12) Dinsheim bei Molsheim (F, Dép. Bas-Rhin): $\pm a .1007$ Dunginisheim; a. 1182 Tunguene sin, a. 1188 Tungenesheim; a. 1320 Düngensheim; a. 1371 Dungesheim; a. 1471 Dingsheimb; mda. Dinse <*T(h)unginis-haima (Cl auss 1895, S. 253; B arth 1960/63, S. 279; R eichsl and III, S. 223).

Die Entwicklung der Ausgangsform frk. *T(h)unglnis-haima ,Siedlung des Thungins' ist folgendermaßen zu skizzieren. Der spätbezeichnete U mlaut des 9. J ahrhundert [u] $>$ [y], der ahd. Wandel von germ. [p] $>$ [d] (anlautend im Oberdeutschen später auch oft mit $<t>$ geschrieben) erzeugt die Form *Düngines-heim, die spätahd. Abschwächung der Endsilbenvokale führt zu *Düngins-, Düngens-; die Assimilation von [ns] > [s] zu *Dünges-; Synkope des unbetonten Endsilbenvokals zu *Düngs-; die Entrundung des [y] zu *Dings-, woraus sich mit weiterer Assimilation von [gs] > [s] und gelegentlich weitergehender Senkung von [i] $>$ [e] die heutigen amtlichen Formen Dinzheim, Dinsheim, Dingsheim und die mundartlichen Resultate Dingse, Dense ergeben. Die überlieferten Formen, zum großem Teil nur kopial überliefert, sind R eflexe dieser Entwicklungsstadien der Siedlungsnamen. J edoch lassen die frühen Belege mit $<t>$ in allen drei Siedlungsnamen nur den Schluss zu, dass die romanisierte fränkische Form tungin(us), tunchin(us) mit romanischem Lautersatz [t] für [p] ebenfalls in den Anfängen präsent war.

Einzelne Verschriftungen sind bemerkenswert. So lässt sich bei $\mathrm{Nr} .11$ die Form Tunchinasheim von a. 788 nur durch einen romanischen Schreiber der Urkunde oder ihrer Vorlage erklären: $<t>$ ist - wie erwähnt eine in romanischem Sprachgebiet häufige Umsetzung des Spiranten [ $p]$, $<c h>$ hindert die vor [i] im Romanischen normale spirantische Aussprache von [g], <ai > in -haim hält archaisierend die sonst längst erloschene germ. Form des Grundworts fest. Auch die merkwürdige W iedergabe des ahd. Genetivs auf -is, -es durch <-as > ist eine Eigenart dieser Urkunde (Wigridashaim, Hantscohasheim, auch Gaganhaim statt *Gagin- und Hughilahaim). Dagegen ist bei Nr. 12 die Form a. 1192 Tunguenesin mit < gu > statt < ge > zur Sicherung des Verschlusslautes 
(Tenuis) und romanischem $\mathrm{h}$-Verlust in *-hen < -hein Zeuge der mundartlichen Entwicklungsform, die mit -in verschriftet wird.

Für die elsässischen drei $* T(h)$ unglnes-haima gibt es nur eine Erklärung auf der Basis der Amtsbezeichnung, da ein Personenname *Thungin nicht existiert. Die drei Siedlungsnamen sind nicht nur Belege für das Amt des Vorstehers des mallus in der ,Lex Salica', sondern auch, da das Amt schon im späteren 6. J ahrhundert zu verschwinden scheint, für den frühen und intensiven fränkischen Einfluss auf das Elsass, das Land der Ali-sătjon, der ,im Ausland Sitzenden', das dann bald, unter dem austrasischen König Childebert a. 589, als fränkische K önigslandschaft aufscheint (L angenbeck 1967, II, S. 58-88; P ol enz 1961, S. 198f.).

\section{Reliktwortschatz in Ortsnamen}

Das Reliktwort Sabel, Savel ,Sand, Sandboden' < rom. *sabulu(m) begegnet nur in den rheinischen Dialekten und in den Varietäten des Niederländischen noch sporadisch. Rudolf Post hat in seiner Arbeit über rheinische Entlehnungen aus dem Romanischen die Verbreitung des Wortes kenntnisreich behandelt (P ost 1982, S. 113, siehe Karte 1 im Anhang).

An die niederländische Zone von savel, das so bereits im Mittelniederländischen belegt ist, schließt sich eine grenznahe niederfränkische und mittelfränkische Zone an. Es begegnet auch im Rheinengtal, an Mosel, Saar und in der Westeifel, in Luxemburg und Lothringen, schließlich gar noch in der anschließenden Westpfalz resthaft. Vor kurzem konnte es auch literarisch um die Mitte des 15. J ahrhunderts als sabel m. (auch in der Doppelformel sant und sabel) in der Bedeutung ,Gries, grobkörniger Sand' in der nach Lothringen zu setzenden, westrheinfränkischen metrischen Übersetzung der ,Pilgerschaft des träumenden Mönchs' des Guillaume de Digulleville nachgewiesen werden. Das Reliktwort ist ein typisches ,W estwort', dessen Verbreitung nie über den engeren deutsch-romanischen K ontaktraum und die Umgebung der Sprachgrenze hinausreichte. Innerhalb dieser Zone gelingt es aber, durch die Saarbrücker Datenbank der Flurnamen des Saarlandes und des germanophonen Lothringens, die Dichtigkeit der Belege im nördlichen Saarland und vor allem in Lothringen noch erheblich zu steigern (Haubrichs 2002, S. 563 mit Abb. 43/44, siehe $\mathrm{K}$ arte 2).

Das Wort findet sich massiert rund um Thionville/Diedenhofen, ebenso dicht im Raum zwischen Nied und Deutscher Nied rund um den Warndt, schließlich auch südlich davon im nördlichen Seillegau (Saulnois), 
dünnt aber gegen Saarbrücken und zur oberen Saar bei Sarrebourg hin aus. Einzelbelege aber legen auch für den Saarbrücker Raum und den oberen Saargau eine ehemals stärkere V erbreitung nahe.

Die Etymologie mit dem Ansatz von lat. sabulu(m) bzw. einer Nebenform *sabellu(m), grobkörniger Sand' wird auch durch diese Belege nicht in F rage gestellt, allenfalls kann an der unmittelbaren Sprachgrenze auch direkte Ableitung von frz. sable erwogen werden, doch spricht insgesamt die auf die frühen Kontaktgebiete (Moselromania, Rheinengtal, Luxemburg, Lothringen) beschränkte Verbreitung eher für frühe Entlehnung (Christmann 1938, S. 15; M ül l er/Frings 1968, S. 447; Ditt maier 1963, S. 254).

Eher ein ,Nordwort' ist das Westgerm. *hais-ja-, *haisipi ,junger Baum, Niederwald'. Für *haisja- bieten niederländische Ortsnamen einige recht alte Belege (K ünzel /B I okN er hoeff 1988, S. 487):

(13) H ees, Gde. Soest (NL, Utrecht): a. 838 kop. 11. J h. in Hesi.

(14) a. 838 kop. Ende 11. J h. in pago Leomeriche...in Wrestarhesi (in den Liemers, NL, Gelderland). Als $\mathrm{H}$ ees und $\mathrm{H}$ eest sind beide Wörter in niederländisch-nordwestdeutschen Ortsnamen bezeugt (vgl. mnd. hese, hes ,Buchenwald'). In nhd. H eister m. (aus älterem *haistru) ist *hais- mit dem Baumnamensuffix *-tru weitergebildet worden (vgl. mhd. heister, mnd. hester, nndl. hei ster).

Die Bedeutung dieser "charakteristisch fränkisch-sächsischen Kennwörter" (H aubrichs 1996, S. 571f.; 1998, S. 109, 111; vgl. 1999, S. 129f.) wurde schon von Theodor F rings und Walther von W ar tbur g 1937 erkannt. Sie wurden auch aus dem Altniederfränkischen ins F ranzösische als hesse ,Buchenwald, Niederwald' bzw. hêtre ,Buche', afrz. hestre übernommen. Die Verbreitung beider Lehnwörter deckt sich nahezu vollständig mit dem westfränkischen Siedlungsraum ( $\mathrm{F}$ r ings/W ar t bur g 1937, S. 204, 207 mit Karte 2, siehe K arte 3 im Anhang).

Im Französischen erscheinen die Wörter sowohl in Ortsnamen als auch im appellativen Wortschatz, wobei frz. hêtre die R esultate von lat. fagus im Norden verdrängte. In der Germania ist $H$ ees(t) nur noch in Flurund Siedlungsnamen bezeugt, während Heister auch noch appellativ, besonders im Dialekt- und Fachwortschatz vorkommt. In Ortsnamen ist $\mathrm{H}$ eister bis Oberhessen, als Appellativum bis zu den Mittelgebirgen belegt (Trier 1952, S. 105; D it t maier 1963, S. 107). Nördlich dieser Zone gilt das Wort im gesamten Niederdeutschen, nicht aber in Schleswig; im transalbingischen Holstein wird es in der ersten $\mathrm{Hälfte}$ des 20. J ahrhunderts als veraltet gemeldet ( $\mathrm{F}$ rings/W ar t bur g 1937, S. 200).

Bemerkenswert ist, dass beide Wörter nach Süden zu den gesamten frühen fränkischen Raum ausfüllen, nicht aber den mittelrheinischen 
Raum südlich Mainz und nicht die ostfränkischen Mainlande. Dies lässt sich nicht zuletzt durch die Belege des saarländisch-lothringischen Flurnamenarchivs belegen. Beide Wörter klingen, so erweist sich, im Saar-Mosel-R aum aus (siehe K arte 4 im Anhang).

Dabei füllt $\mathrm{H}$ ees nahezu das ganze Lothringen und das ganze Saarland aus und setzt sich auch nach W esten in Waldnamen wie H esse fort. W iederum geben aus Flur- und Waldnamen hervorgegangene Siedlungsnamen willkommene ältere B elege, die bis ins 7. J ahrhundert zurückreichen:

(15) Hesse (F, Dép. Moselle, Ct. Sarrebourg): a. 699 kop. 9. J h. ad Chassus, var. Cassus (mit rom. Lautersatz für germ. [ ] anzeigenden Schreibungen $\langle c h\rangle_{,}\langle c\rangle$ ); a. 801 kop. 9. J h. in villa que dicitur Essem (mit rom. h-Aphaerese); a. 846 kop. 9. J h. in Essi; a. 847 kop. 9. J h. Hessis; a. 1049 kop. in loco qui dicitur Hesse. Bei den aus W eißenburger Überlieferung stammenden $F$ rühbelegen ist romanischer Lautersatz [a] für den germ. Diphthongen [ai] festzustellen.

(16) † H eysberg, Gde. Distroff (F, Dép. Moselle, Ct. Metzervisse): a. 1309 in loco qui dicitur H eysberch; F lurname mda. H eisbrich <*haisja- +*-berga- m. ,Berg'.

Die $\mathrm{K}$ arte zeigt auch, dass $\mathrm{H}$ eister nicht so weiträumig in Namen verbreitet ist, sondern sich in Anlehung an das von Heinrich Dittmaier skizzierte Verbreitungsgebiet (vereinzelt Hunsrück, Eifel, Neuwied, Altenkirchen, Nassau, B ergisches Land und Südniederfränkisch) im nördlichen Saarland und dann südlich bis an die lothringische Nied. Dittmaier wies auch hier bereits bis ins 12. J ahrhundert zurückreichende Belege nach ( $D$ it t maier 1963, S. 107):

(17) H eisterschoß (Siegkreis): a. 1129 H eistersaz ,J ungwaldschösslinge'.

(18) a. 1184 bei K leve: Scarpheistere ,Schmitt-H eister'.

(19) a. 1248 bei Düsseldorf, Kr. M ettmann: Eychheister ,Eichen-J ungwald“.

(20) a. $1315 \mathrm{im} \mathrm{K} \mathrm{reis} \mathrm{Bernkastel} \mathrm{a.} \mathrm{d.} \mathrm{Mosel:} \mathrm{zuschent} \mathrm{dem} \mathrm{H} \mathrm{eister.}$

(21) a. 1569 in Aachen-R eichsbüsch: die H eisteren.

(22) a. 1580 in Roxheim (K r. K reuznach): im H eister.

Die Belege im Saarland und an der lothringischen Nied setzen bereits im elften $\mathrm{J}$ ahrhundert ein und liefern damit anscheinend einen Erstbeleg:

(23) † H eistrebach, Gde. Téting-sur-Nied ( $F$, Dép. Moselle, Ct. Faulquemont/F alkenberg): a. 1018 or. Heistrebach (MGH DD Heinrich II, Nr. 379) < thais-tru + westgerm.*-baki,Bach'.

(24) † H eisterbach, Gde. Nalbach (Kr. Saarlouis): $\pm a .1344$ kop. um 1400 zu Heisterbach; $\pm a$. 1340/50 kop. um 1400 H eysterbach.

(25) Heisterberg, Gde. Namborn (Kr. St. Wendel): a. 1379 or. zu Heisterberg; a. 1383 or zuo H eysterberg <*hais-tru + germ. *-berga- m. ,Berg'. 
Es scheint also, dass das auch frz. hêtre, afrz. hestre zugrundeliegende *hais-tru sich aus einem wohl eher westlich gelegenen Kerngebiet - der Erstbeleg ist zugleich auch der westlichste Beleg im germanophonen $\mathrm{Ge}$ biet - weiterverbreitet hat.

\section{Straß enbezeichnungen in Sied I ungsnamen und $\mathrm{F}$ I urnamen}

Die Flurnamen sind voll von alten Wege- und Straßenbezeichnungen (H aubr ichs 1997, S. 154ff.), die in wenigen Fällen auch zu Siedlungsnamen geworden sind, wie etwa im Falle von Langmeil, a. 1534 die Lange Mille (D ol ch/G r eul e 1991, S. 277f.), eine metonymische Straßenbezeichnung mîle, z. B. a. 1685 gemeine Landstraße, die lange M eile genannt für die Rheintalstraße von Köln bis Godorf. Darunter sind viele auf Grund gewandelter kultureller und technischer Bedingungen ausgestorbene oder selten gewordene Appellative; manche von innen gehören zur frühesten Schicht volkssprachiger Straßenwörter: so das seit karolingischer Zeit als Lehnübersetzung oder Aequivalent von strata publica oder via publica aufscheinende Appellativum diotweg (zu ahd. diot < germ. *theuda- ,Volk, gens, populus'). Auch ahd. heristrâza, ae. herestraet ,H eerstraße' zu *harja'Schar, Heer' war vielleicht ursprünglich Aequivalent für via publica im Sinne von, öffentliche Straße'. Schon das a. 786 (?) bei Dorndorf am Werraübergang der sogenannten ,Kinzigstraße' nach Eisenach mit platea que dicitur Hohastraza belegte Wort Hoch-Straße bezeichnete eine mit erhöhtem Straßendamm gebaute, wohl oft auf römischer Grundlage liegende Straße, oder auch in anderen Fällen eine über die Höhen verlaufende, die sumpfigen Niederungen meidende Straße. Die aus Namen rückzugewinnenden, aber noch mhd. lebendigen Wörter Rennweg, Rennstraße, a. 822 Renniphat (zu ahd. rennan, schnell laufen, reiten') galten für über die Höhen verlaufende Schnellstraßen. Der für eine via pavata, eine gepflasterte Straße gebrauchte Ausdruck war ahd. steinweg, as. stênweg (um 840/50 im altsächsischen Bibelepos ,Heliand') oder steinstrâze, später auch am deutschen W estrande als Lehnwort Pavei. Einer vielleicht nicht mehr karolingischen Lehnschicht gehört als Übersetzung von via regis an die Bezeichnung kuningesweg, -strâze (a. 980 bei Fulda in K uningesweg). Noch später, und zwar zuerst nicht etwa in einer Urkunde oder einer Rechtsquelle, sondern in einer Dichtung, im um 1200 entstandenen Artusroman ,I wein' des Hartmann von Aue erscheint dann die uns so geläufige und kaum hinterfragte, in ihrer Genese aber wohl an die Entstehung von Landesherr- 
schaft und der von ihr ausgehenden Strukturierung des L andes gebundene Bildung landstrâze.

Vielleicht eine der interessantesten Bezeichnungen findet sich in dem appellativ nahezu ausgestorbenen, zwischen Mosel und Rhein verbreiteten, aus gallolat. camm $n$ nus (frz. chemin), Straße' abzuleitenden und in mehreren Varianten vorliegenden Lehnwort K emmen, K emmel, Kimmel, Kümmel bzw. in Kurzform $(\mathrm{Kem}(\mathrm{m}), \mathrm{Kim}(\mathrm{m})$. Das Wort findet sich in Flurnamen im R heinland, in Luxemburg, in Lothringen, im Saarland und in der Pfalz (Vannér us 1936; D it t maier 1963, S. 137f.; Christ mann 1943; Niedeer ehe 1967, S. 40ff.; H er bil I on 1968, S. 81-86; Frings/M ül I er 1966, I, S. 68; II:, S. 146; Hal fer 1988, S. 252, Nr. 626; H aubrichs 1997, S. 107ff.). Nach der Entlehnung wurde die aus der rom. Form *camminu entstandene Lehnform *camín durch den ahd. i-U mlaut (8. J h.) zu *kemin umgelautet. In mittelhochdeutscher Zeit hat sich dann durch Endsilbenabschwächung kemen entwickelt:

(26) SN † K emmen bei Helfant (Kr. Trier-Saarburg): a. $1279 \mathrm{~K}$ emmen.

(27) a. 1317 prope viam dictam K emene bei Loertzange (Luxemburg).

(28) a. 1460 uf den K emen bei Bernkastel an der Mosel.

Die Weiterentwicklung zu kemel lässt sich auf partielle Dissimilation Wechsel $[n]>[l]$ wegen vorausgehenden Nasals - zurückführen. Der Erstbeleg findet sich in:

(29) SN † K emel bei Langenschwalbach (Rheingau-Taunuskreis): 1011 Kamele, 1250 camel (mit nicht bezeichnetem U mlaut).

Daneben tritt auch eine voll an das ehemalige [i] der Endsilbe assimilierte Variante kimmel auf, vorwiegend in der Pfalz:

(30) a. $1434 \mathrm{~K}$ ymelgaß in St. J ohann bei Hornbach (K r. Zweibrücken).

(31) für die römische R heinuferstraße bei R heinzabern (Kr. Germersheim): a. 1599 der K imel; a. 1598 Tümmel item K ümmel (gerundete Variante).

Die Kurzformen $\mathrm{Kem}(\mathrm{m}), \mathrm{Kim}(\mathrm{m})$ lassen sich durch die Verlagerung des Akzentes auf die erste Silbe bei fortschreitender Integration ins Althochdeutsche erklären, ferner durch die Synkopierung des Endsilbenvokals, der zu einer Angleichung des auslautenden [-n] an das im Wortzentrum stehende [-m-] führte. Das Wort ist rezent noch als lothr. $K$ em m./n. (z. B. im Gen. Kemptjes We), lux. Kiem (u. a. auch Kiemt, Kiemech, Kiemel, Kiemert, z.B. hochkiemert ,hohe Straße', hi erkemet ,H eerstraße', M etz kimmert ,Straße nach Metz') (F oll mann 1909, S. 282; Lux. Wörterbuch II, S. 346). Die Flurnamen weisen, wo es sich erkennen lässt, auf maskulines 
Genus des Wortes hin. Der Erstbeleg als Hodonym, als Wegename scheint zu sein:

(32) a. 1317 superior via que dicitur K eme für die Römerstraße Metz-Trier.

Das in Flurnamen massenhaft belegte Wort war zweifellos früher auch als Appellativ weiter verbreitet. Die F lurnamenbelege sind geradezu Indizien für die ehemalige Extension des Wortes. Für die Bedeutungsermittlung kann die erhaltene appellative Geltung in Lothringen und Luxemburg Hinweise geben. Es bezeichnet dort Fernstraßen von hohem Alter, dabei sicherlich oftmals, aber keineswegs immer römische Straßen, was in der Forschung allzu häufig übersehen wird. Auch Flurnamen-Belege wie hochkem in U ckange, chemin de haut kem und hautkem in Grandage, a. 1606 auf dem kehmet, aber rezent sur les chaussee des romains in Roussy-le-V illage, steinkehm in Bouss (alle im Dép. Moselle) nähren den Eindruck, dass es sich hierbei um variierende Bezeichnungen für alte Fernwege handelt. $\mathrm{K}$ emen, $\mathrm{K}$ emel war auf alle $\mathrm{F}$ älle ein universales Straßengrundwort mit der Bedeutung, die schon lat. camm $\llbracket$ nus hatte. Es ist deshalb wie Straße und Weg zusammensetzungsfähig gewesen: Man kann dafür vergleichen bei den Flurnamen steynkemen, steinkemel oder auch auf dem ellen kiem, Eylenkehm (zu einem Lehnwort aus lat. olla ,Topf'), uf dem hilzen K emen ,der Holzbohlenstraße', hochkiemert ,Hochstraße', M etz kimmert ,Metzer Straße', hierkemet ,Heerstraße', der Prümmer K ehmen ,Prümer Straße' usw.

Das Saarbrücker ,Archiv der Siedlungs- und Flurnamen des Saarlandes und des germanophonen Lothringen' (ASF SL) konnte die bisher bekannte Anzahl der B elege für $\mathrm{K} \mathrm{em}(\mathrm{m}) \mathrm{el}, \mathrm{K} \mathrm{em}(\mathrm{m})$ etc. erheblich vermehren (siehe Karte 5 im Anhang).

Die areale Distribution der diversen Lautformen hat im Saar-MoselRaum ihren Schwerpunkt im nördlichen Lothringen um Thionville/Diedenhofen und Sierck, also im unmittelbar an Luxemburg anschließenden Gebiet; so verdichten sich die Belege auch im nordwestlichen moselnahen Saarland. Daneben finden sich Belege in lockerer Streuung in den Gebieten, in denen das Appellativ nicht mehr lebendig ist, südlich bis etwa zur $\mathrm{H}$ öhe Sarrebourg an der oberen Saar. Die intensiver aufgenommenen toponymischen Belege des Saar-Mosel-Raums bestätigen, dass es sich um ein Wort vorwiegend der Trierer und Metzer Diözese mit einem vorgelagerten Feld an der alten R heingrenze handelt.

Die bereits phonetisch behandelte Aufspaltung der Resultate von *camm[nu in Kurz- und Langformen lässt sich eventuell durch zwei Entlehnungsschichten erklären. Die Kurzformen $\mathrm{K}$ eim, $\mathrm{K}$ em, $\mathrm{K}$ iem, $\mathrm{K}$ im etc. des Wegewortes finden sich vor allem im intensivsten germano-romani- 
schen Interferenzgebiet, vorwiegend entlang der Sprachgrenze. So stammen aus Luxemburg etwa die Belege a. $1317 \mathrm{~K}$ eme bei Grevenmacher, a. 1601 lanset die K eme bei Stehnen, a. 1615 zwischen den zweyen $\mathrm{K}$ heime bei Barnich, a. 1647 sur le Kim bei Aubange (V annér us 1936, S. 285f., 288). Hier ist wohl von einer frühen fränkischen Entlehnungsstufe auszugehen, deren Charakteristikum eben die erwähnte auslösende Akzentkonzentration auf der ersten Silbe darstellt. Eine zweite Entlehnung dürfte sich bei anhaltender bilingualer Sprachsituation in der Moselromania noch vor dem althochdeutschen i-Umlaut (8./9. J h.) und vor der romanischen Palatalisierung von [ka] (10. J h.?) entwickelt haben.

\section{Concl usio}

Gemeinsam ist den drei skizzierten Themenbereichen der Beziehungen zwischen Appellativen und Toponymen, denen viele andere zur Seite zu stellen wären, dass die in innen behandelten $W$ örter in eine außerordentlich frühe Zeit, in die Zeit des frühen Mittelalters zurückreichen. Ortsnamen bergen frühe Amtsbezeichnungen wie bischof ,Bischof', kuning ,König', auch ausgestorbene wie kuningāri ,Königsleute', vor allem aber das in der ,lex Salica' belegte fränkische Rechtswort thung[n, bald aussterbend, aber doch durch sein Vorkommen in elsässischen Toponymen die Expansion der Franken des 6. J ahrhunderts ins Elsass bezeugend. Ortsnamen enthalten in den germano-romanischen Interferenzgebieten um Trier und Metz frühe agrarische Lehnwörter wie sabel, sawel, grober Sand, Sandboden' und viele andere. Ortsnamen bezeugen die frühe Extension von nördlich, im fränkischen und sächsischen Raum verbreiteten Agrarwörtern wie thais-ja ,junger Baum, Niederwald" und *hais-tru ,junger Baum, Buche' und bezeugen auch ihre nur im frühen Mittelalter, in der Kontaktsituation des Galloromanischen und des Fränkischen mögliche Expansion nach Nordfrankreich. Ortsnamen helfen bei der Rekonstruktion des frühen Straßen- und Wegewortschatzes, so in der germanischromanischen K ontaktzone des Saar-Mosel-R aums der aus rom. *cammদnu herzuleitenden W egebezeichnungen kemen, $\operatorname{kemel}, \operatorname{kem}(\mathrm{m})$.

Die vorgeführten Wörter belegen aber in ihrer Gesamtheit noch mehr als die Möglichkeit der Rekonstruktion von frühem Wortschatz aus Toponymen. Sie belegen, dass frühe romanische Lehnwörter ins Fränkische diffundierten, frühe fränkische Lehnwörter aber mit der Expansion der Franken wanderten, ins Elsass, in das Romanische Nordfrankreichs, in das lateinisch kodifizierte Recht der Franken sogar. So belegen die Orts- 
namen letzten Endes, wenn auch mittelbar, dass wir für die Zeit des frühen Mittelalters, für die Zeit vor dem Festwerden der Sprachgrenze nicht mit ,reinen', säuberlich getrennten Sprachsituationen zu rechnen haben. Die Sprache der Franken, die Sprache der Romanen in diesem großen Interferenzraum waren aufnahmebereite, aufnahmefähige, aus Bilingualität heraus sich neuformierende Sprachen.

\section{Bibl iographie}

Al br echt, Karl (Hrsg., 1891/98).: Rappoltsteinisches Urkundenbuch 759-1500. Quellen zur Geschichte der ehemaligen Herrschaft Rappoltsstein im Elsass, 5 Bde. Colmar.

Barth, Medard (1960/63): Handbuch der elsässischen Kirchen im Mittelalter. Straßburg (Archives de l'Eglise d'Alsace 27-29).

Bruckner, Albert (1949): Regesta Alsatiae aevi Merovingici et Karolini (496-918), Bd. 1: Quellenband. Straßburg, Zürich.

Christmann, Ernst (1938): Beiträge zur Flurnamenforschung im Gau Saarpfalz. München, Berlin (Die Flurnamen Bayerns, R eihe IX, Untersuchungen 1).

Chr ist mann, Ernst (1943): « K em, K im, Kümmel»als Benennungen für R ömerstraßen von Luxemburg-Metz bis Speyer-Lauterburg. In: Germania. Anzeiger der Römisch-Germanischen K ommission 27, S. 72-79.

Cl auss, J oseph M. B. (1895): Historisch-topographisches Wörterbuch des Elsass, Bd. 1. Zabern.

D it t maier , Heinrich (1963): R heinische F lurnamen. Bonn.

D ol ch, Martin/G r eul e, Albrecht (1991): Historisches Siedlungsnamenbuch der Pfalz. Speyer.

Förstemann, Ernst (1913/16): Altdeutsches Namenbuch, Bd. II: Die Ortsnamen. 3. Aufl., hrsg. v. Hans J el I inghaus. Bonn.

F ol I mann, Michael Ferdinand (1909): Wörterbuch der deutsch-lothringischen Mundarten. Metz.

F rings, Theodor/W ar tbur g, Walther von (1937): Französisch und Fränkisch. In: Zeitschrift für Romanische Philologie 57, S. 193-210.

GI öckner, Karl/D ol I, Anton (1979): Traditiones Wizenburgenses. Die Urkunden des K losters W eissenburg 661-684. Darmstadt.

$\mathrm{H}$ al $\mathrm{fer}$, Manfred (1988): Die F lurnamen des oberen Rheinengtals. Ein Beitrag zur Sprachgeschichte des Westmitteldeutschen. Wiesbaden, Stuttgart (Mainzer Studien zur Sprachund Volksforschung 12).

$\mathrm{H}$ aubr ichs, Wolfgang/R amge, Hans (Hrsg., 1983): Zwischen den Sprachen. Siedlungs- und Flurnamen in germanisch-romanischen Grenzgebieten. Saarbrücken (Beiträge zur Sprache im Saar-Mosel-R aum 4).

H aubr ichs, W olfgang (1996): Sprache und Sprachzeugnisse der merowingischen F ranken. In: Die Franken - Wegbereiter Europas, Bd. 1, Mannheim, S. 559-573.

Haubrichs, Wolfgang (1997): Die volkssprachlichen Bezeichnungen für alte Fernwege im Deutschen, vorwiegend nach westmitteldeutschen Quellen dargestellt. In: Friedhelm Burgar d, Alfred Haver kamp (Hrsg., 1997): Auf den Römerstraßen ins Mittelalter. Bei- 
träge zur Verkehrsgeschichte zwischen Maas und Rhein von der Spätantike bis ins 19. J ahrhundert (Trierer Historische Forschungen 30), S. 97-181.

H aubrichs, Wolfgang (1998): Fränkische Lehnwörter, Ortsnamen und Personennamen im Nordosten der Gallia. Die ,Germania submersa' als Quelle der Sprach- und Siedlungsgeschichte. In: Dieter Geuenich (Hrsg., 1998): Die Franken und die Alemannen bis zur „Schlacht bei Zülpich“ (496/97) (Ergänzungsbände zum Reallexikon der Germanischen Altertumskunde 19). Berlin, New Y ork, S. 102-129.

H aubrichs, Wolfgang/St ein, Frauke (1999): Frühmittelalterliche Siedlung im Saarbrücker Raum. In: Rolf W it tenbr ock (Hrsg., 1999): Geschichte der Stadt Saarbrücken, Bd. 1. Saarbrücken, S. 111-158; 623-632.

H aubrichs, Wolfgang (1999): Sprachliche Differenzen und Kongruenzen zwischen Sachsen und Franken innerhalb der ,Westgermania'. In: H.-J. H äß I er (Hrsg., 1999): Sachsen und Franken in Westfalen. Zur Komplexität der ethnischen Deutung und Abgrenzung zweier frühmittelalterlicher Stämme. Oldenburg (Studien zur Sachsenforschung 12), S. $123-142$.

H aubrichs, W olfgang (2002): Die Pilgerfahrt des träumenden Mönchs. Eine poetische Übersetzung Elisabeths aus dem Französischen? In: Wolfgang H aubrichs, Hans-Walter H er r mann (Hrsg., 2002): Zwischen Deutschland und Frankreich. Elisabeth von Lothringen, Gräfin von Nassau-Saarbrücken. St. Ingbert (Veröffentlichungen der Kommission für Saarländische Landesgeschichte und Volksforschung 34), S. 533-568.

$H$ aubr ichs, W olfgang (2003): Thung[n, K uning, M eistar. Amtsbezeichnungen in elsässischen Siedlungsnamen des frühen Mittelalters. In: Peter Thor au, Sabine Penth, Rüdiger Fuchs (Hrsg., 2003): Regionen Europas - Europa der Regionen. Festschrift für KurtUlrich J äschke zum 65. Geburtstag. Köln, Weimar, Wien, S. 7-19.

H enzen, Walter (1957): Deutsche W ortbildung, 2. Aufl. Tübingen.

H er bil I on, J ules (1968): R outes et chemins en toponymie gallo-romaine. In: Revue Belge de Philologie et d'Histoire 46, 1, S. 81-86.

$K$ auf mann, Henning (1976): R heinhessische Ortsnamen. Die Städte, Dörfer, Wüstungen, Gewässer und Berge der ehemaligen Provinz Rheinhessen und die sprachgeschichtliche Deutung ihrer Namen. München.

K ünzel, R. E./BI ok, D. P.N erhoeff, J . M. (1988): Lexicon van nederlandse toponiemen. Amsterdam.

Langenbeck, Fritz (1967): Studien zur elsässischen Siedlungsgeschichte. Vom Weiterleben der vorgermanischen Toponymie im deutschsprachigen Elsass, 2 Bde. Bühl.

M eid , W olfgang (1966): Die Königsbezeichnung in den germanischen Sprachen. In: Die Sprache 12, S. 182-189.

M ül I er , Gertraude/F r ings, Theodor (1966): Germania R omana, 2 Bde. Halle.

Nieder ehe, Hans-J osef (1967): Straße und Weg in der galloromanischen Toponomastik. Genf, Paris.

Pol enz, Peter von (1961): Landschafts- und Bezirksnamen im frühmittelalterlichen Deutschland. Untersuchungen zur sprachlichen R aumerschließung. Marburg.

Post, Rudolf (1982): Romanische Entlehnungen in den westmitteldeutschen Mundarten. Diatopische, diachrone und diastratische Untersuchungen zur sprachlichen Interferenz am Beispiel des landwirtschaftlichen Sachwortschatzes. Wiesbaden (Mainzer Studien zur Sprach- und Volksforschung 6).

Das R eichsland Elsass-Lothringen. Landes- und Ortsbeschreibung, Teil III. Straßburg 19011903. 
Ramge, Hans (Hrsg., 1987): Hessischer Flurnamenatlas (Arbeiten der Hessischen Historischen Kommission Neue Folge, Bd. 3). Darmstadt.

R amge, Hans (2002): Hrsg. Südhessisches F lurnamenbuch. Darmstadt.

Reitzenst ein, Wolf-Armin Freiherr von (1986): Lexikon bayerischer Ortsnamen. Herkunft und Bedeutung. München.

Sittler, Lucien (1958): Les listes d'admission à la bourgeoisie de Colmar (1361-1494). Colmar.

Sch midt -W iegand, Ruth (1972): Fränkische und frankolateinische Bezeichnungen für soziale Schichten und Gruppen in der Lex Salica. In: Nachrichten der Akademie der Wissenschaften in Göttingen, Phil.-Hist. KI. 1972, Nr. 4, S. 219-259. Neu in: Dies.: Stammesrecht und Volkssprache. Ausgewählte Aufsätze zu den Leges barbarorum. Weinheim, S. 355-391.

Schmidt -W iegand, Ruth (1989): Die Malbergischen Glossen - eine frühe Überlieferung germanischer Rechtssprache. In: Heinrich Beck (Hrsg., 1989): Germanische Rest- und Trümmersprachen. Berlin, New Y ork, S. 157-174.

Schmidt-W iegand, Ruth (1998): Thunginus. In: Handwörterbuch der Rechtsgeschichte, Bd. 5, Sp. 213-216.

Tr ier , J ost (1952): Holz. Etymologien aus dem Niederwald. Münster, Köln.

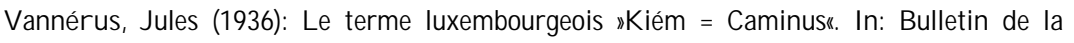
Commission Royale de Toponymie et de la Dialectologie 10, S. 277-332.

W eitzel, J ürgen (1985): Dinggenossenschaft und Recht. Untersuchungen zum Rechtsverständnis im fränkisch-deutschen Mittelalter, Bd. 1. K öln, Wien.

W eit zel , J ürgen (1992): Mallus, mallum. In: Lexikon des Mittelalters, Bd. 6, Sp. 175.

W enskus, Reinhard (1986): Bemerkungen zum thunginus der Lex Salica. In: Ders.: Ausgewählte Aufsätze zum frühen und preußischen Mittelalter. Hrsg. v. Hans P at ze. Sigmaringen, S. 65-84. 


\section{ANHANG}

\section{$\underline{K}$ artenlegenden}

1) sabel m. ,Gries, grober Sand' < lat. sabellum

Quelle: R. Post, Romanische Entlehnungen in westmitteldeutschen Mundarten, Wiesbaden 1982, K arte 24.

2) sabel m. Sand' in rezenten und historischen Flurnamen des Saar-M osel-R aums

3) Verbreitung der Abkömmlinge von westgermanisch *haisja, haisi pi, *hais-tru Quelle: Th. Frings/W. v. Wartburg, ZrPh 57 (1937), S. 207 Karte 2.

4) H ees und $\mathrm{H}$ eister in F lur- und Siedlungsnamen des Saar-Mosel-R aums

5) $\mathrm{Kem}(\mathrm{m}) \mathrm{el}, \mathrm{K}$ em(m) etc. <lat. camm凹nus, Weg, Straße' in F lurnamen des Saar-MoselRaums 


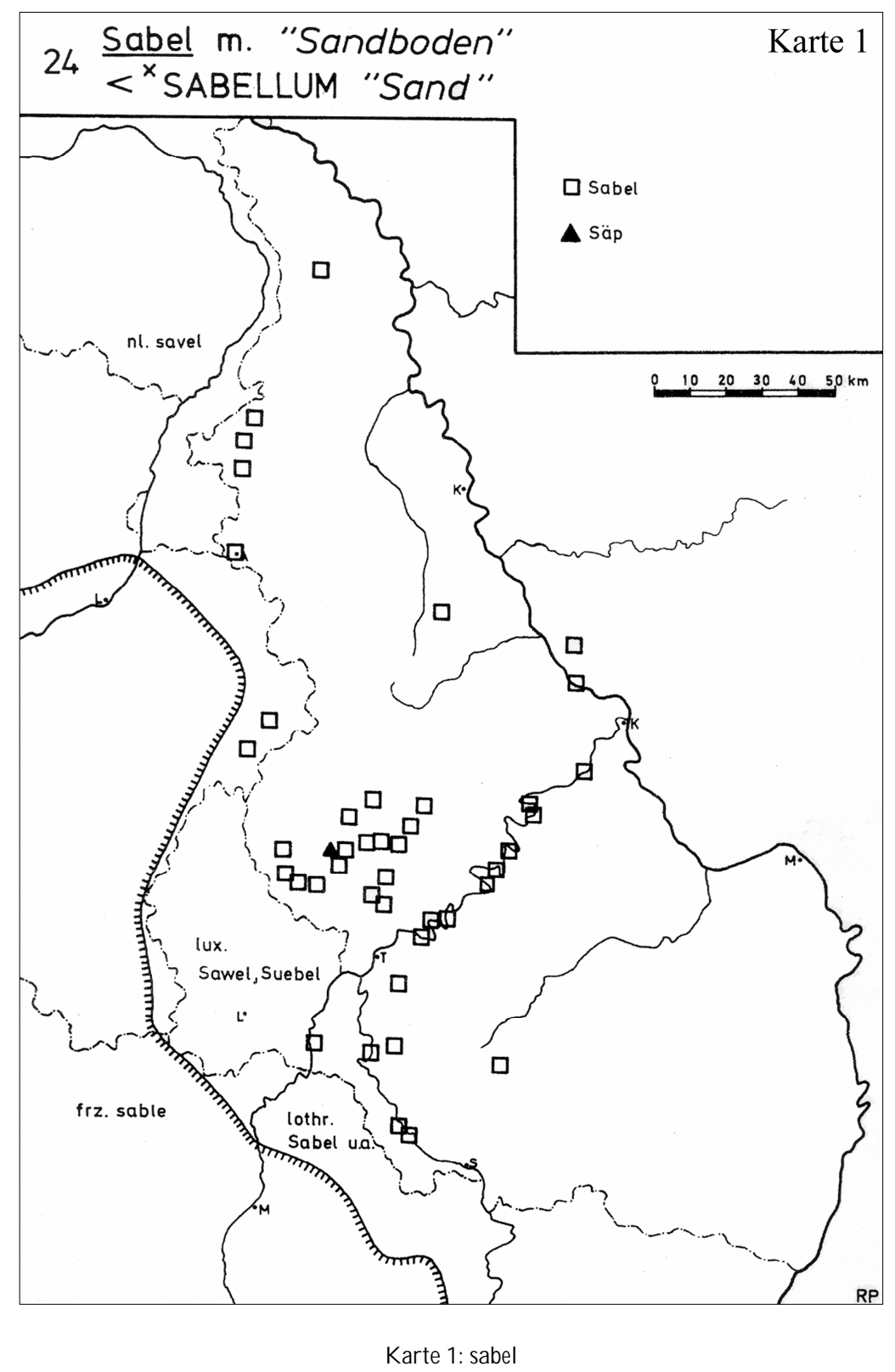




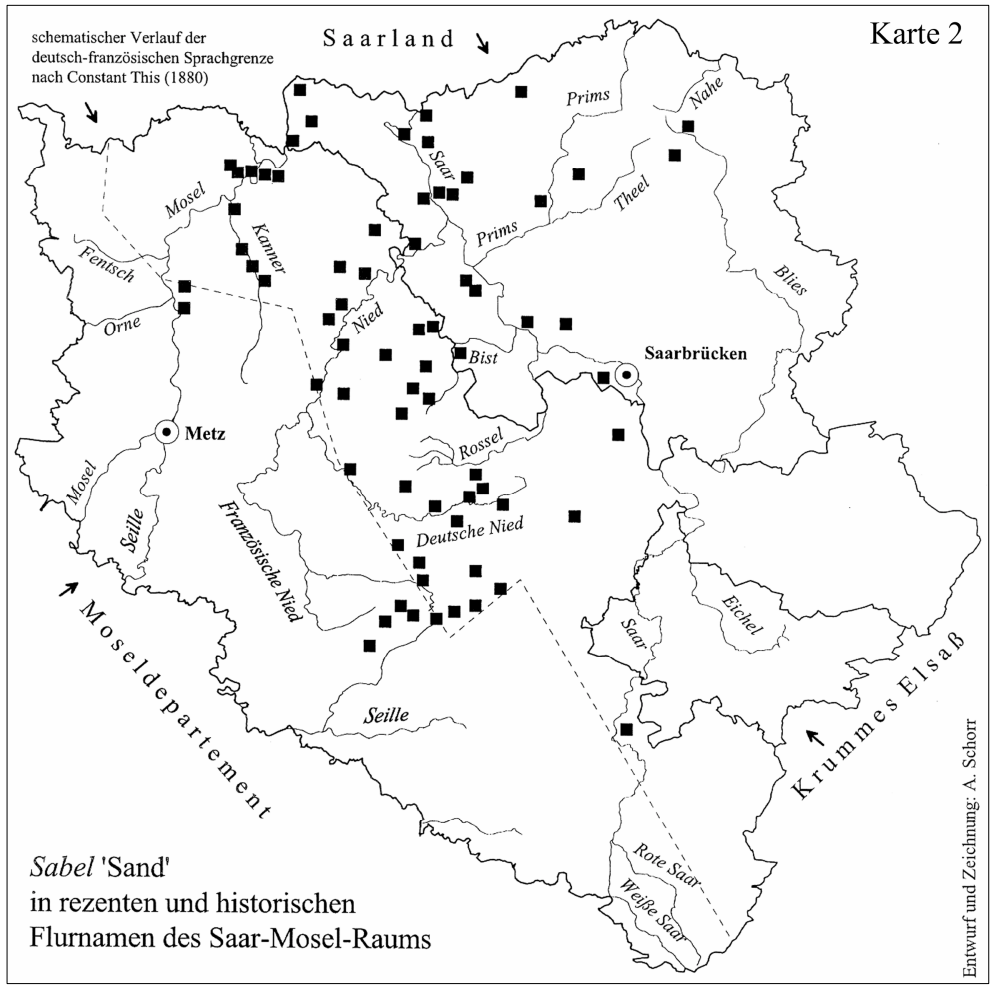

Karte 2: sabel in rezenten und historischen Flurnamen des Saar Mosel-Raums 


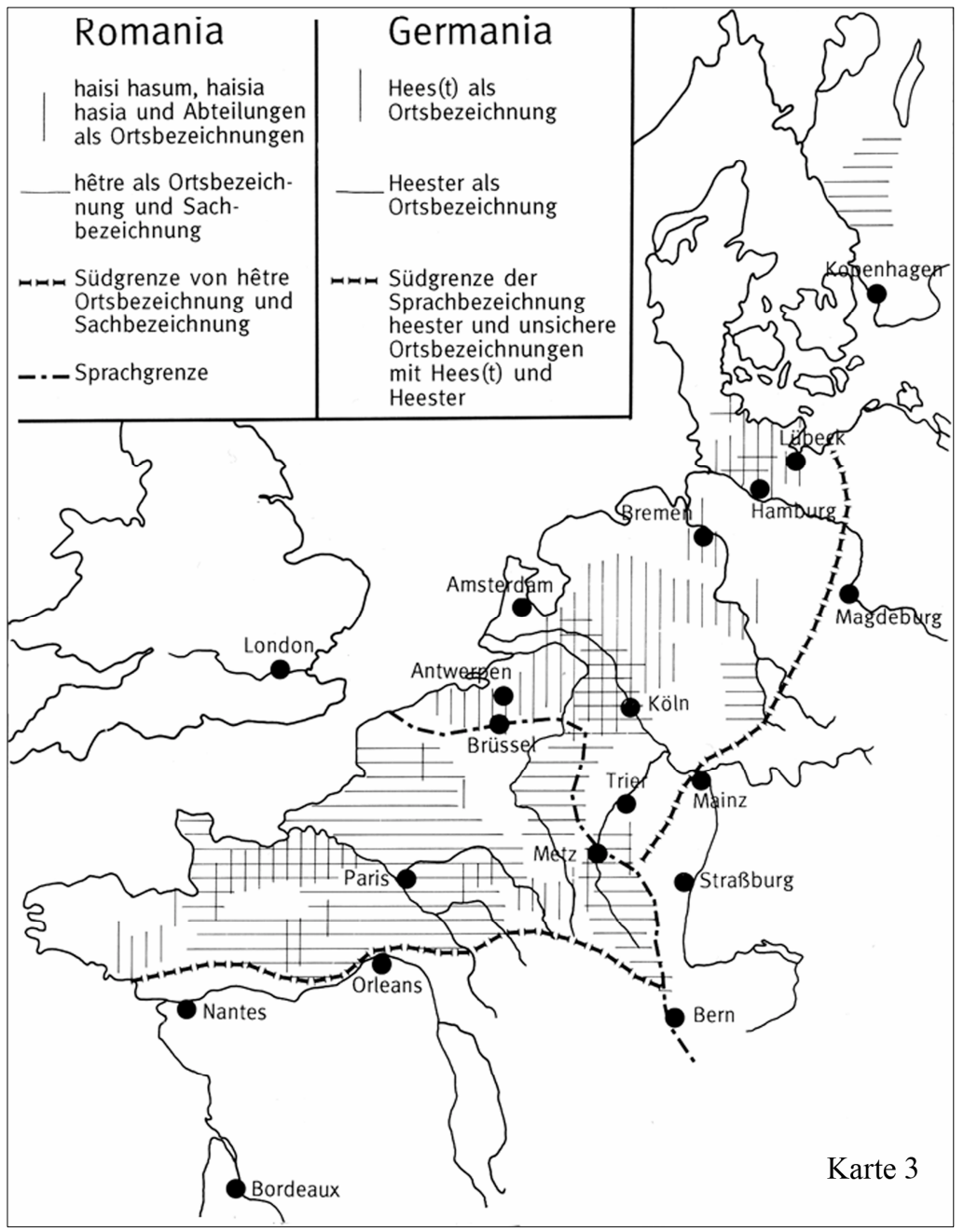

Karte 3: Verbreitung der Abkömmlinge von westgermanisch *haisja, haisi pi, *hais-tru 


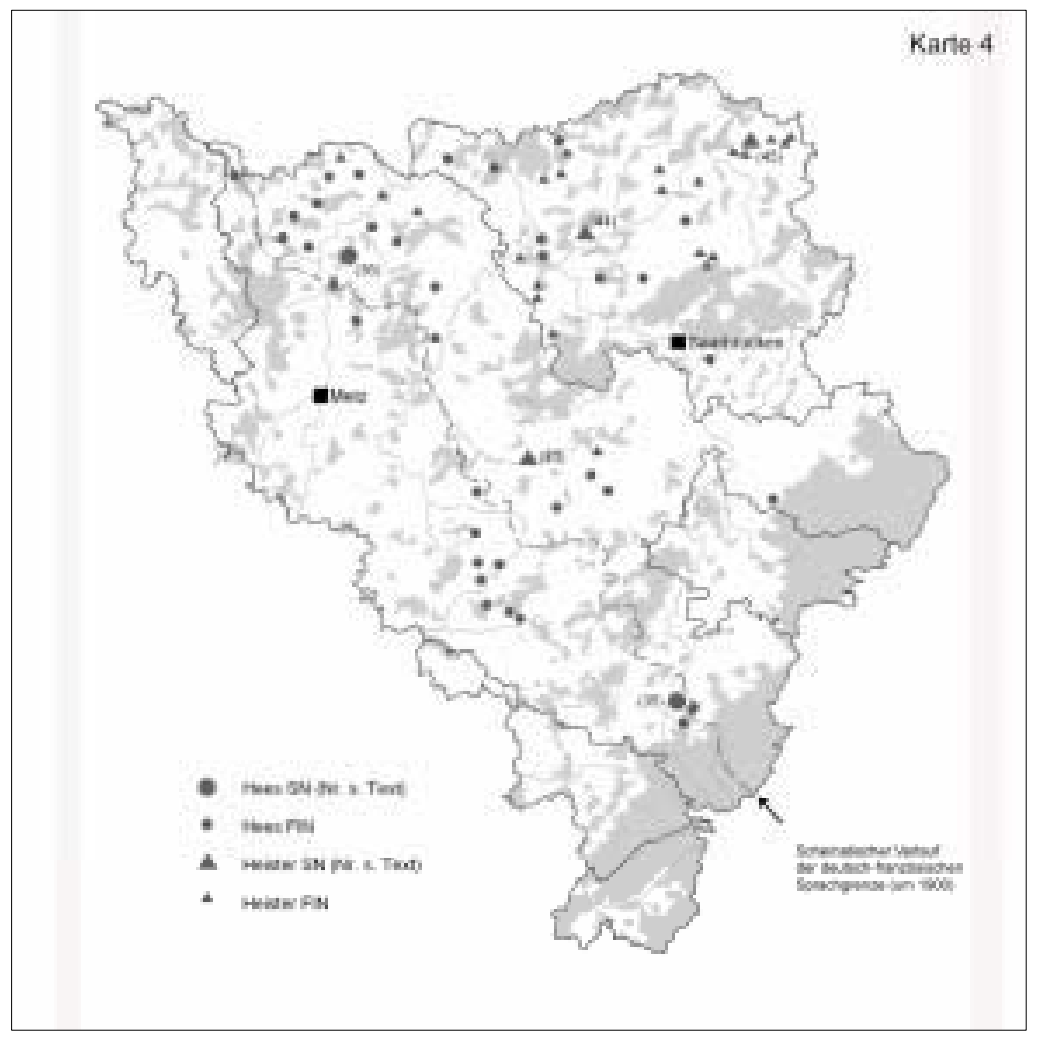

Karte 4: $\mathrm{H}$ ees und $\mathrm{H}$ eister in F lur- und Siedlungsnamen des Saar-M osel-R aums 


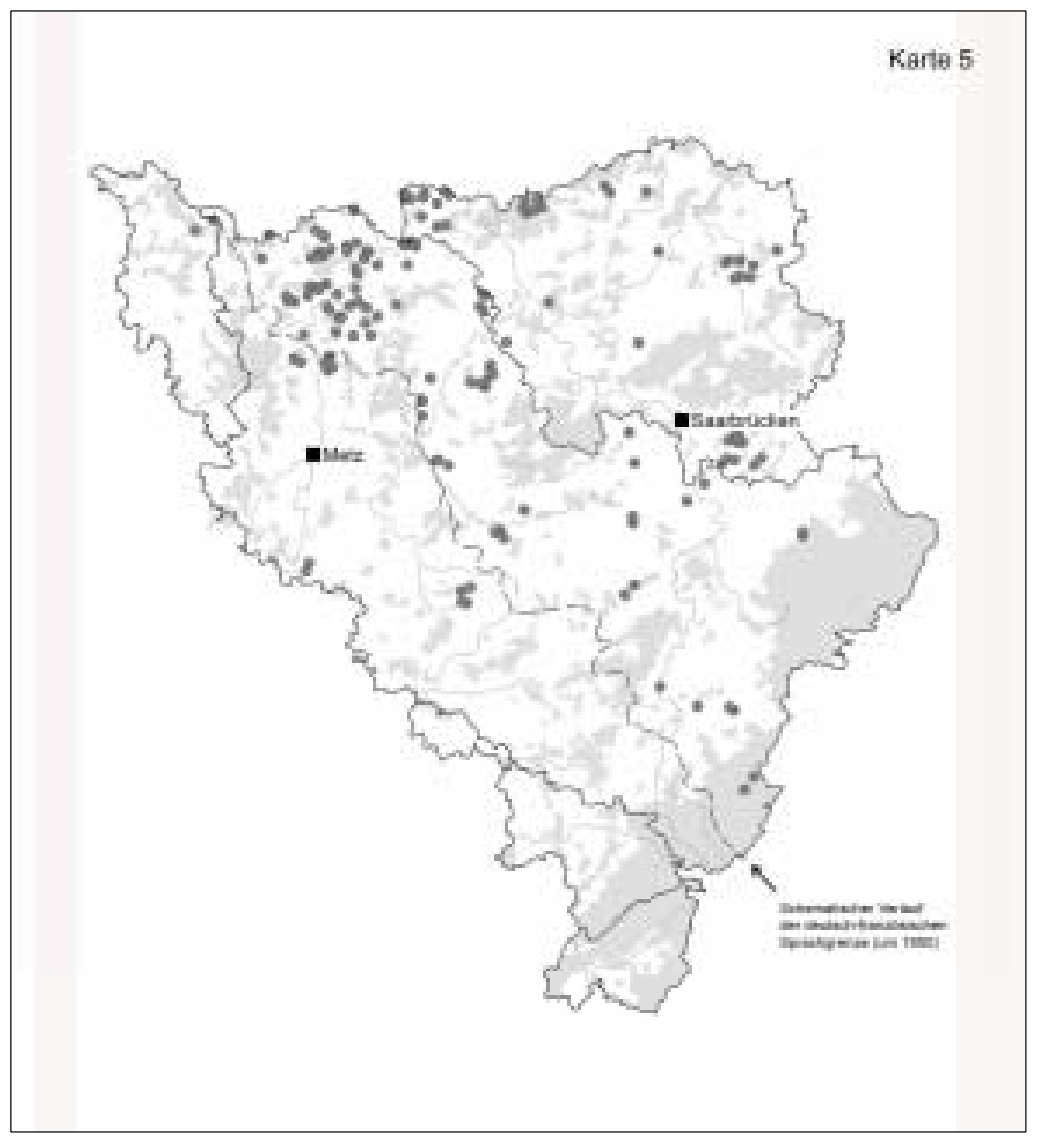

$\mathrm{K}$ arte 5: $\mathrm{K}$ em(m)el in F lurnamen des Saar-Mosel-R aums 
